\title{
On the multi-frequency emission and evolution of the white dwarf binary system AR Scorpii
}

\section{P.J. Meintjes*t}

Department of Physics, University of the Free State, Po Box 339, Bloemfontein, South Africa

E-mail: MeintjPJeufs.ac.za

\begin{abstract}
The peculiar close binary system AR Scorpii, since its discovery in 2016, sparked huge interest due to its peculiar multi-frequency emission from radio frequencies to X-ray energies. The multi-frequency Spectral Energy Distribution (SED) from this system is dominated entirely by non-thermal emission from radio to X-ray energies, which resulted in several theoretical papers appearing in literature that attempted to explain the peculiar multi-frequency properties of this enigmatic source. In this paper, an emphatic theoretical framework will be presented to explain the multi-frequency SED of AR Sco, as well as a model to explain the possible origin and evolution of this enigmatic source.
\end{abstract}

XII Multifrequency Behaviour of High Energy Cosmic Sources Workshop

12-17 June, 2017

Palermo, Italy

*Speaker.

${ }^{\dagger}$ A footnote may follow. 


\section{Introduction}

The discovery of the close binary system AR Scorpii (AR Sco) exhibiting very significant multi-frequency pulsed emission form radio to optical frequences ([1]) sparked huge interest in the astronomical community (see Fig. 1). The system consists of a 0.8-1.29 $M_{\odot}$ white dwarf orbiting a $0.28-0.45 M_{\odot}$ M5 secondary star with an orbital period of $P_{\text {orb }}=3.56$ hours. These estimates are based on the fact that the secondary is a Roche lobe filling star, based on ellipsoidal variations of the optical light curve (see Fig.2). However, the lack of evidence of mass transfer may imply that the secondary may be slightly detached. The distance to the source is approximately $d \sim 116 \mathrm{pc}$ ([1]).

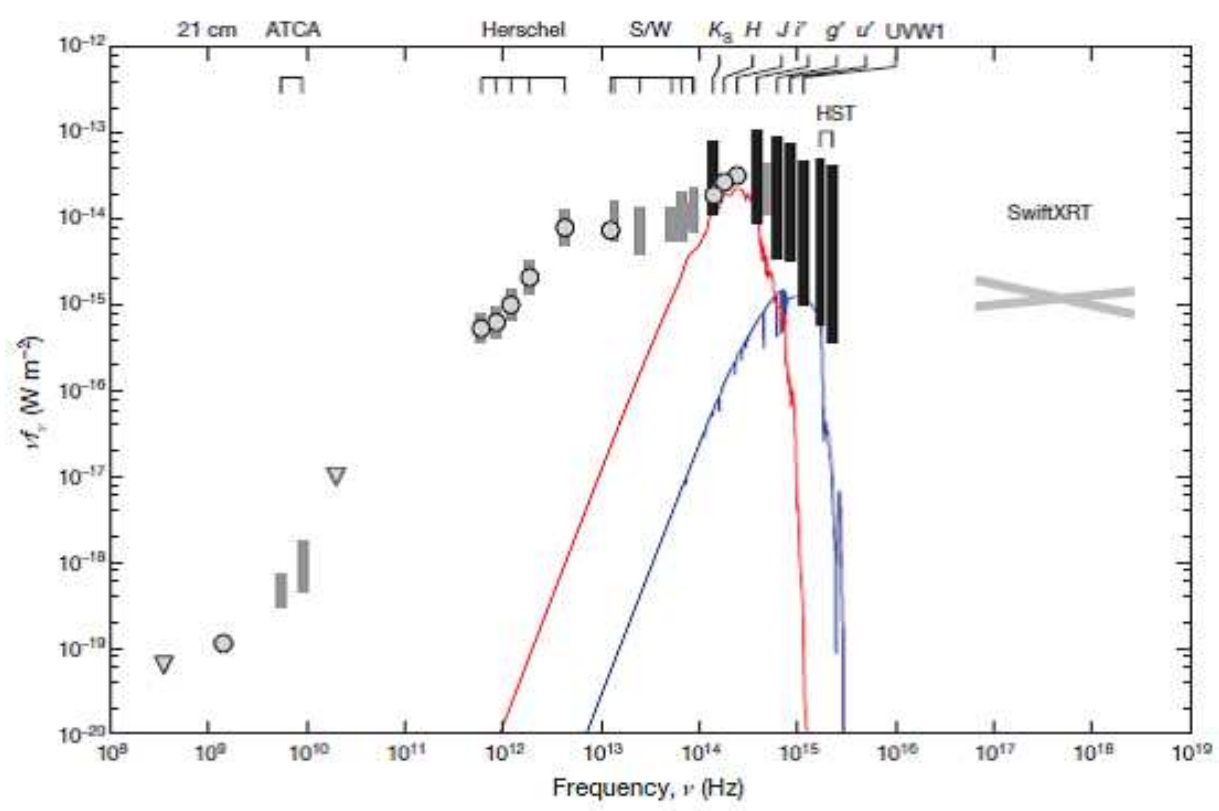

Figure 1: The multi-frequency Spectral Energy Distribution (SED) of AR Scorpii from radio to X-ray frequencies. The red and blue curves represent model atmospheres of the red M5 secondary dwarf star $(\mathrm{T}=$ $3100 \mathrm{~K})$ and white dwarf $(\mathrm{T}=9750 \mathrm{~K})$ respectively. Adopted from [1].

Closer investigation (e.g. [1]) of the pulsed emission superimposed on the orbital modulation, reveals strong pulsed emission at a period of of $P_{\mathrm{B}}=1.97 \mathrm{~min}(118.2 \mathrm{~s})$, which is a beat period between the white dwarf spin period of $P_{\mathrm{s}}=1.95 \mathrm{~min}(117.1 \mathrm{~s})$ and the orbital period of the binary $P_{\text {orb }}=3.56 \mathrm{hr}$. Low resolution optical observations performed at the UFS-Boyden Observatory outside Bloemfontein (SA) illustrate the double-pulse nature of the periodic modulation in the period range around the spin and beat periods of the white dwarf (see Fig. 3). This double pulse implies emission and consequential pumping of the secondary from both polar caps of the spinning white dwarf. This double pulse is clearly visible in a periodogram, revealing the double pulse rotation and beat periods at the fundamental and first harmonic (see Fig. 4). The beat period is more prominent at the first harmonic around $17 \mathrm{mHz}$, where two distinct pulses are observed, namely the spin frequency (on the right) and the beat (on the left). 


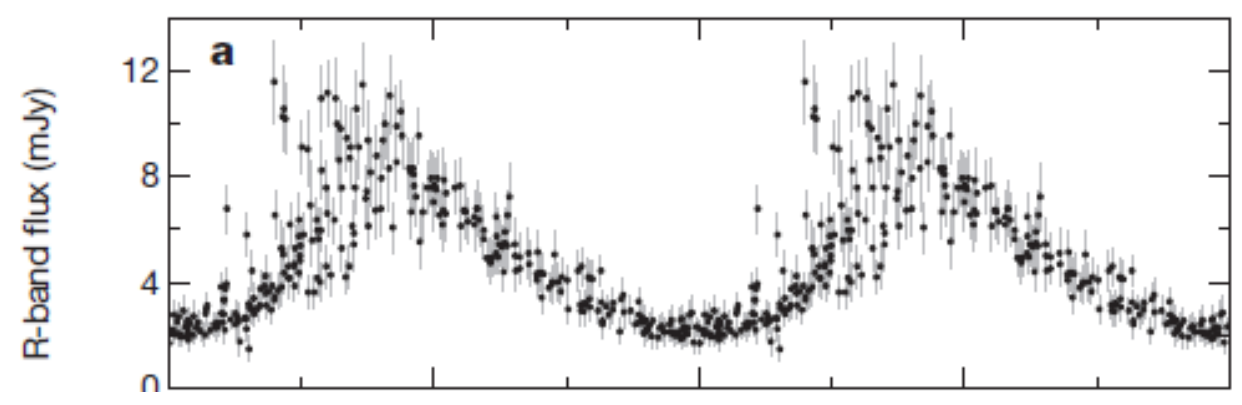

Figure 2: The orbital modulation from AR Scorpii in R-band, showing ellipsoidal variations that may be due to a Rochle lobe filling (or nearly lobe filling) secondary star. Superimposed on this orbital light curve, optical pulsations can be seen, the nature of which wil be discussed shortly. Adopted from [1].

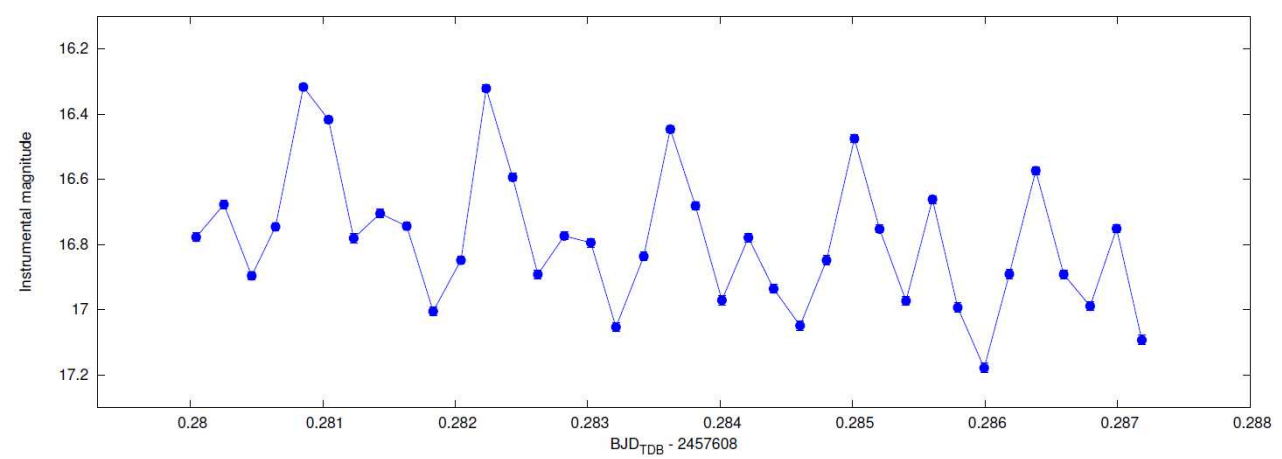

Figure 3: The system shows strong double pulsed emission close to the spin period of the white dwarf, illustrated by this low resolution optical light curve that was obtained from optical observations performed using the UFS-Boyden $1.5 \mathrm{~m}$ telescope equipped with an Apogee U 55 CCD camera at the Boyden Observatory outside Bloemfontein, South Africa. Data supplied by Me. H. Szegedi (M.Sc Student)

Recent high-speed all-Stokes optical polarimetry of AR Sco ([2]), utilizing the South African Astronomical Observatory (SAAO) HIPPO polarimeter ([3]) on the $1.9 \mathrm{~m}$ telescope, reveals strong linear polarization of the double pulse emission at levels up to $40 \%$, with significantly lower levels of circular polarization $(<10 \%)([2]$.

Pulse timing studies over a baseline of 7 years ([1]) show that the white dwarf is slowing down, resulting in a frequency derivative $-\dot{v}_{\mathrm{s}, \mathrm{wd}}=2.86 \times 10^{-17} \mathrm{~Hz} \mathrm{~s}^{-1}$, which for a $\sim 0.8 M_{\odot}$ white dwarf $\left(R_{\mathrm{wd}} \approx 0.01 R_{\odot}\right)$, infer in a spin-down luminosity of approximately $\left.L_{\dot{\mathrm{v}}_{\mathrm{s}, \mathrm{wd}}} \approx 1.5 \times 10^{33} \mathrm{erg} \mathrm{s}^{-1}[1]\right)$.

Another peculiar property of AR Sco is the ratio of the X-ray to spin-down luminosity of the white dwarf, i.e. $L_{\mathrm{x}} \sim 10^{-3} L_{\dot{\mathrm{s}}_{\mathrm{s}, \mathrm{wd}}}$, similar to the white dwarf in the nova-like variable system AE Aquarii ([4]). This places both these white dwarf binary systems in the same class as the spin-powered pulsars ([5]) (see Fig. 6). This implies that the multi-frequency emission in both these sources are driven by the spin-down of the white dwarf and not by mass accretion from the secondary star. The observed spin down of the white dwarf in AR Sco allows an upper limit to be placed on the magnetic field strength of the white dwarf if one infers that magnetic dipole radiation 


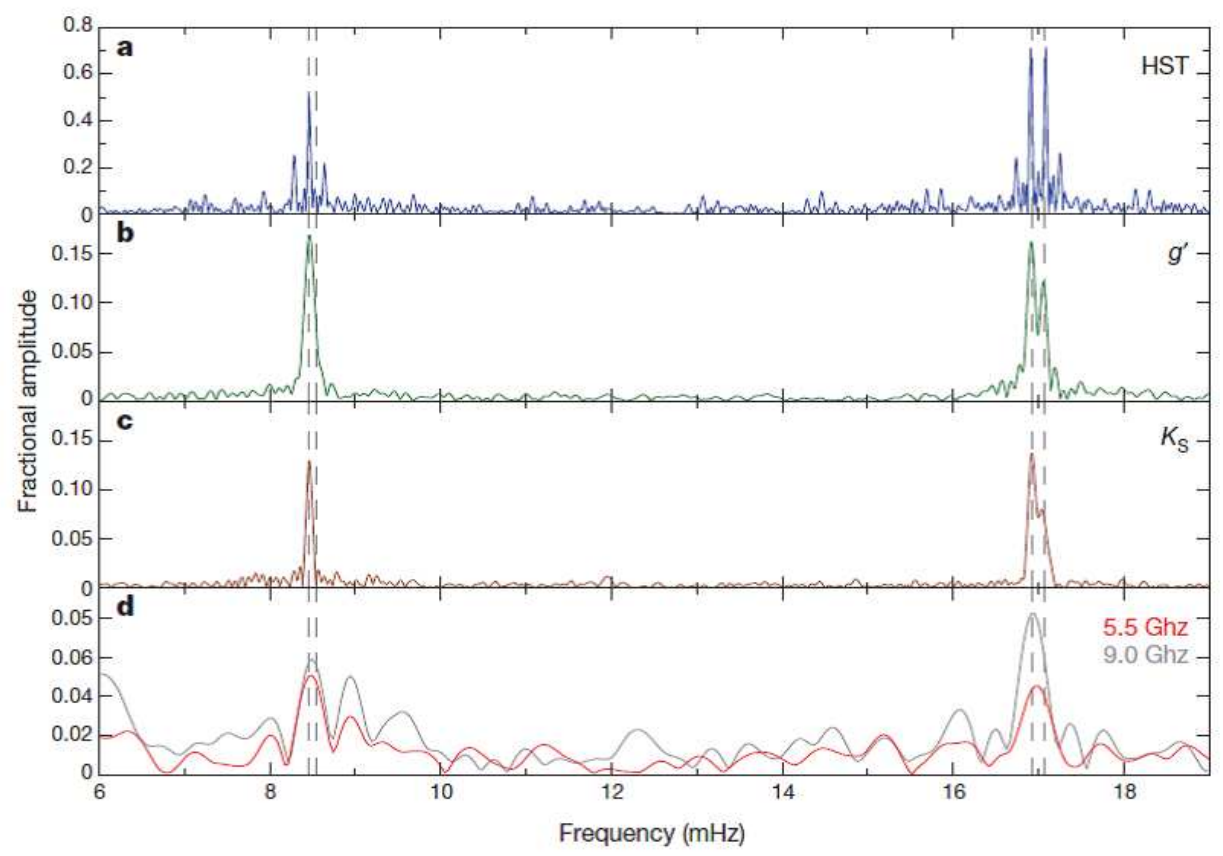

Figure 4: Periodograms from radio to optical (HST), all revealing the strong double pulse beat period between the spin and orbital periods of the system. The beat pulse and the spin frequency are most prominent at the first harmonic, close to $17 \mathrm{mHz}$. The peak on the left represents the beat while the peak on the right represents the spin frequency. Adopted from [1].

(e.g. [6]) alone drains the rotational kinetic energy. Based upon this assumption, it can be shown (see [2]) that the magnetic field upper limit for AR Sco is

$$
B_{1, *} \sin \chi=\left(\frac{3 c^{3} L_{\dot{v}_{\mathrm{s}}} P_{\mathrm{wd}}^{4}}{2(2 \pi)^{4} R_{\mathrm{wd}}^{6}}\right)^{1 / 2},
$$

which results in (assuming a magnetic inclination angle $\chi=90^{\circ}$ )

$$
B_{1, *} \leq 500\left(\frac{L_{\dot{v}_{\mathrm{s}}}}{1.5 \times 10^{33} \mathrm{erg} \mathrm{s}^{-1}}\right)^{1 / 2}\left(\frac{P_{\mathrm{wd}}}{117 \mathrm{~s}}\right)^{2}\left(\frac{R_{\mathrm{wd}}}{5.5 \times 10^{8} \mathrm{~cm}}\right)^{-3} \mathrm{MG} .
$$

Based on the orbital separation (see [2]) between the highly magnetic white dwarf and secondary star, the magnetic field of the white dwarf at a distance equal to the binary separation, i.e. at a distance equal to the center of the secondary star, is

$$
\begin{aligned}
B_{1, \mathrm{sec}}= & B_{1, *}\left(R_{\mathrm{wd}} / a\right)^{3} \\
& \leq 160\left(\frac{B_{1, *}}{500 \mathrm{MG}}\right)\left(\frac{a}{8 \times 10^{10} \mathrm{~cm}}\right)^{-3}\left(\frac{R_{\mathrm{wd}}}{5.5 \times 10^{8} \mathrm{~cm}}\right)^{3} \mathrm{G} .
\end{aligned}
$$

It is believed that magnetic interaction between this white dwarf field with the coronal fields of the secondary star, plays a very important role in the peculiar multi-frequency emission from this enigmatic source. 

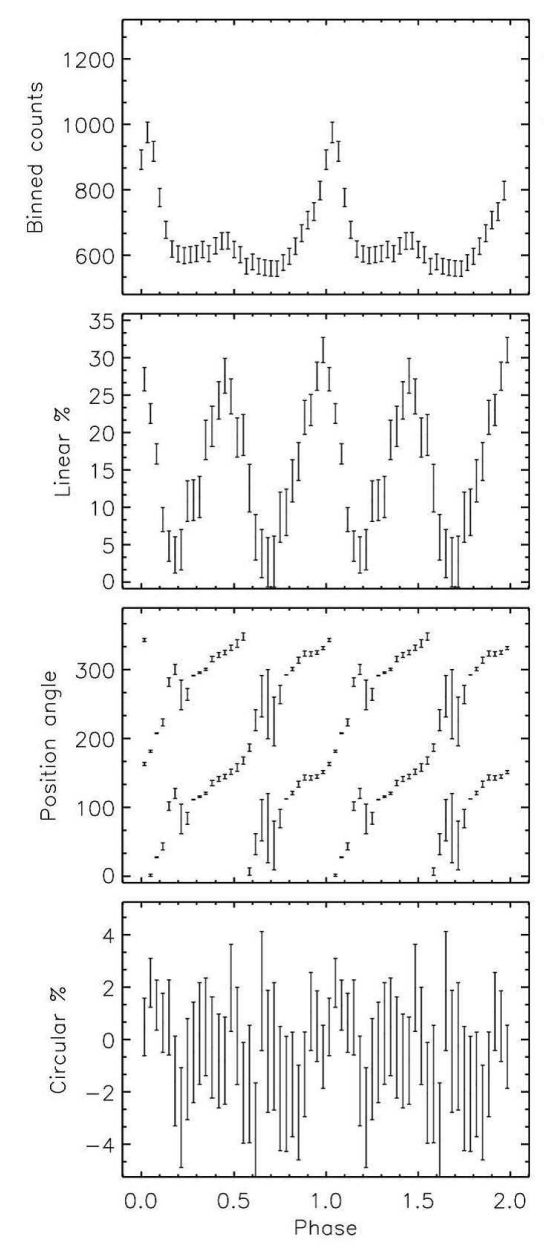

Figure 5: Pulsed linear polarization observed with the HIPPO Polarimeter on the SAAO $1.9 \mathrm{~m}$ telescope at Sutherland (SA). Strong linear polarization of up to $40 \%$ and has been observed, with significantly lower level of circular polarization. The $360^{\circ}$ swing in the position angle is consistent with synchrotron emission produced in the magnetospheric fields of a rotating magnetic dipole, strengthening the notion that AR Sco contains a white dwarf pulsar. Adapted from [2].

The paper will be structured as follows: In the next section a brief discussion of the observed Spectral Energy Distribution (SED) of AR Sco will be presented. In the section following that a model will be presented to explain the multi-frequency SED of AR Sco from radio to X-rays, followed by a brief discussion of the possible evolution of this system and finally, some conclusions.

\section{The Multi-Frequency Spectral Energy Distribution (SED) of AR Sco}

In a recent study ([2]) the Spectral Energy Distribution (SED) of AR Sco (see Fig. 1) has been analysed as a two-component non-thermal spectrum. The first component shows a $v S_{v} \propto v^{1.3}$ slope between $10000 \mathrm{MHz}-5000 \mathrm{GHz}$, resembling a superposition of synchrotron flares produced by expanding synchrotron emitting plasmoids ([7]), similar to the nova-like variable AE Aquarii 


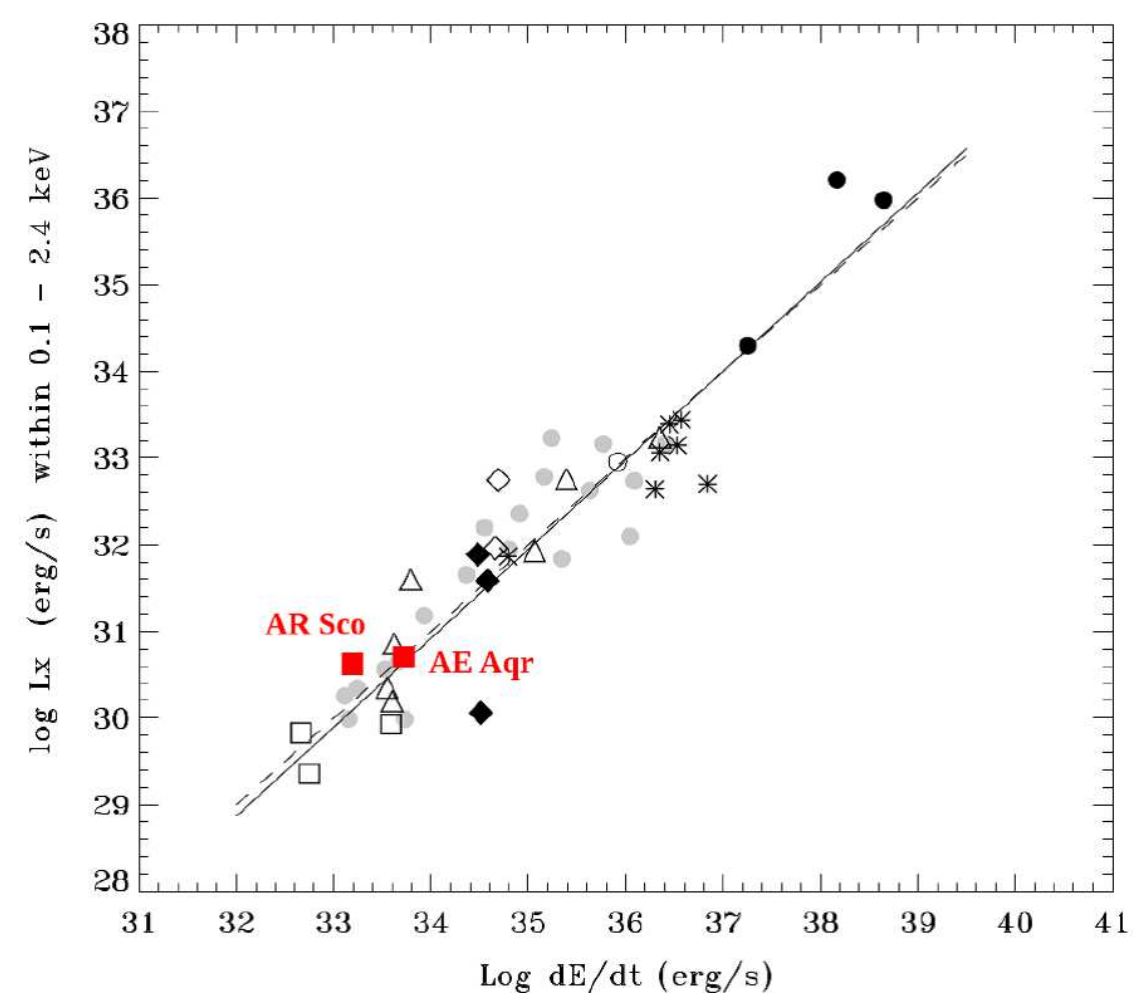

Figure 6: The x-ray luminosity versus spin-down power of spin-powered neutron stars, compared to both the white dwarfs in AR Sco and AE Aquarii. Adapted from [5].

$([8,9])$, while the second component resembles an $v S_{v} \propto v^{-0.2}$ optically thin synchrotron spectrum between $10^{14} \mathrm{~Hz}-10^{18} \mathrm{~Hz}$. The SED (see Fig. 1) strongly suggests that these are separate components produced by different processes and perhaps also in different locations inside the binary system. This implies that the whole SED from radio frequencies to perhaps X-ray energy is produced entirely by the non-thermal synchrotron cooling of relativistic electrons in the binary system, albeit at different locations and driven by different processes. A detailed theoretical framework that could explain the entire SED was presented by ([2]). These processes will be discussed in more detail in the next sections.

\section{Modelling the Multi-frequency SED of AR Sco}

The multi-frequency properties of AR Sco resulted in significant interest since the discovery paper ([1]) was published, with several theoretical papers appearing in close succession attempting to explain the peculiar multi-frequency emission of this system (e.g. [10, 11, 12]). A theoretical framework to evaluate the multi-frequency emission of AE Aquarii was presented in (see [2]), which will be the focus of the discussion in the following section.

\subsection{The Radio-IR SED: Magnetic Pumping of Secondary Star}

The ellipsoidal variations seen in optical light curves suggest that the secondary star in AR Sco 
is filling, or nearly filling, its Roche lobe. This would imply a fast rotating secondary star, whose rotation is phase locked with the binary rotation period, namely $P_{\text {orb }}=3.56$ hour. Recently it has been shown that fast rotating M-dwarf stars can have substantial surface magnetic field strengths in excess of 1 kilo Gauss (e.g. [13]). Furthermore, it has been shown that magnetic braking of secondary stars in close binaries can be a powerful drain of angular momentum that may drive mass transfer in close binary systems ([14]). Based on different classes of dynamo laws (e.g. [15] for a detailed overview), the mass transfer in secondary stars have been modelled for different saturated field (SF) and unsaturated field (USF) dynamo laws. This resulted in estimates for, among other, surface polar magnetic field strength versus orbital period.

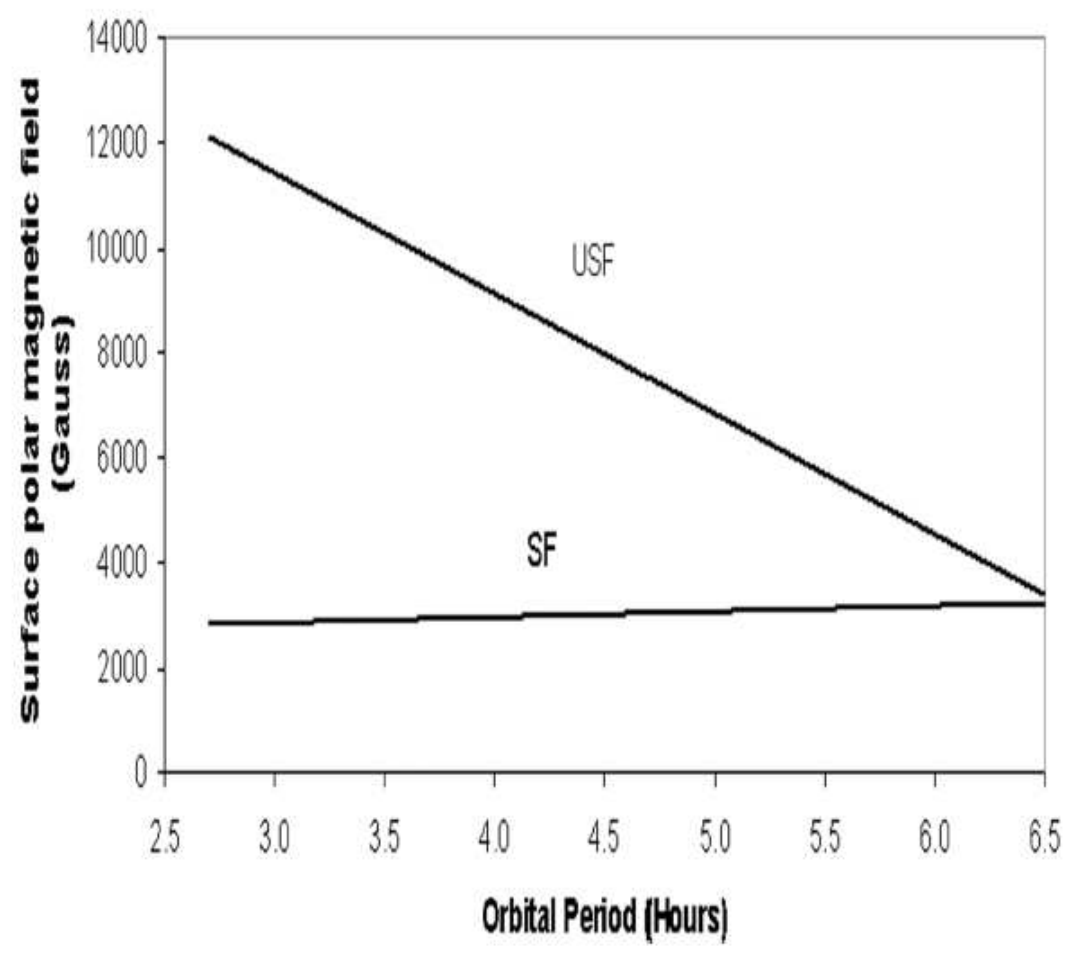

Figure 7: The secondary star surface polar magnetic field strength versus orbital period, based on a Mestel and Spruit wind model for saturated (SF) and unsaturated (USF) fields. Adopted from [16].

It has been shown that the dynamo will saturate for very short rotation periods of the secondary star (e.g. [15]). Incorporating the field saturation, it can be shown that surface polar fields of 3 kilo Gauss ([16]) can be generated, in accordance with the observed values for fast rotating $\mathrm{M}$ dwarfs, quoted by Landstreet \& Donati (i.e. [13]).

Interaction between the fast rotating magnetosphere of the white dwarf and coronal fields of the magnetized secondary star can generate field aligned potentials that accelerate charged particles to high energies. For example, it can be shown that the compression of the secondary star's coronal fields by the white dwarf field sweeping periodically across it, can generate potentials ([17]) of the order of 


$$
\begin{aligned}
\Phi_{\|} & =\left(\frac{300\left(\Delta B_{\perp}\right)^{3}}{64 \pi e n_{\mathrm{e}}^{3 / 2} \sqrt{4 \pi k T}}\right) \mathrm{V} \\
& \sim 200\left(\frac{\Delta B_{\perp}}{700 \mathrm{G}}\right)^{3}\left(\frac{n_{\mathrm{e}}}{10^{10} \mathrm{~cm}^{-3}}\right)^{-3 / 2}\left(\frac{T}{10^{4} \mathrm{~K}}\right)^{-1 / 2} \mathrm{MV}
\end{aligned}
$$

where we assumed $\Delta B_{\perp} \rightarrow B_{1, \mathrm{~L} 1} \sim 700 \mathrm{G}$ represents the maximum perturbation induced in the coronal loops of the secondary star, based on the field strength of the white dwarf at the surface of the secondary star close to the $\mathrm{L} 1$ region.

Potentials of this magnitude can accelerate charged particles like electrons then to energies $\gamma_{\mathrm{e}} \rightarrow 400$, which can result in synchrotron emission in average coronal fields of $\sim 100$ Gauss to frequencies up to

$$
v_{\mathrm{syn}} \sim \gamma_{\mathrm{e}}^{2} e B /\left(2 \pi m_{\mathrm{e}} c\right) \leq 4 \times 10^{13}\left(\gamma_{\mathrm{e}} / 400\right)^{2}(B / 100 \mathrm{G}) \mathrm{Hz}
$$

i.e. in the infrared regime where the observed $v S_{v} \propto v^{\alpha_{1}}\left(\alpha_{1} \approx 1.3\right)$ spectrum. peaks. The continuous pumping of the coronal loops may result in the generation of relativistic electron clouds trapped in the coronal fields, that may result in a superposition of synchrotron flares from these expanding clouds, similar to the radio flares seen in the novalike variable AE Aquarii (e.g. [8,9].

\subsection{Optical-X-ray SED: Pulsar Emission and Striped Wind}

The absence of mass transfer from the secondary star in AR Sco implies a binary system with very low plasma density. If the plasma density in the binary system is low enough, i.e. close to or below the Goldreich-Julian density ([18]),

$$
\begin{aligned}
n_{\mathrm{G}-\mathrm{J}} & =\left(\frac{\Omega_{\mathrm{wd}} \cdot B}{2 \pi e c}\right) \\
& \leq 3 \times 10^{5}\left(\frac{P_{\mathrm{wd}}}{117 \mathrm{~s}}\right)^{-1}\left(\frac{B_{*}}{500 \mathrm{MG}}\right) \mathrm{cm}^{-3},
\end{aligned}
$$

field aligned potentials may grow along the open magnetic field lines cutting through the light cylinder $\left(r_{\mathrm{lc}}\right)$, i.e. where the corotating field has a velocity equal to the speed of light $\left(c=\omega_{\mathrm{wd}} r_{\mathrm{lc}}\right)$. For AR Sco $r_{\mathrm{lc}}=6 \times 10^{11}\left(\omega_{\mathrm{wd}} / 0.054 \mathrm{rad} \mathrm{s}^{-1}\right) \mathrm{cm}$, which implies that the entire binary system $\left(a \sim 8 \times 10^{10}\left(M_{\mathrm{wd}} / 0.8 M_{\odot}\right)^{1 / 3}\left(P_{\mathrm{orb}} / 3.56 \mathrm{hr}\right)^{2 / 3} \mathrm{~cm}\right.$ (e.g. [2]) comfortably fits in the accelerator zone. For AR Sco, it can be shown $([19,2])$ that potentials of the order of

$$
\Delta V \sim 10^{12}\left(\frac{P_{\mathrm{wd}}}{117 \mathrm{~s}}\right)^{-5 / 2}\left(\frac{\mu}{8 \times 10^{34} \mathrm{G} \mathrm{cm}^{3}}\right)\left(\frac{R_{*}}{5.5 \times 10^{8} \mathrm{~cm}}\right) \text { Volt }
$$

can be induced between the white dwarf and the light cylinder. This electric field can produce a relativistic wind of electrons (and ions), with energies of the order of $\gamma_{\mathrm{e}} \sim 10^{6}$, which will emit synchrotron radiation at frequencies up to $v_{\mathrm{syn}} \leq 3 \times 10^{17}\left(B_{\mathrm{lc}} / 0.4 \mathrm{G}\right)\left(\gamma_{\mathrm{e}} / 10^{6}\right)^{2} \mathrm{~Hz}$ (soft X-rays) in the vicinity of the light cylinder radius. Since the whole binary system is inside the accelerator 
zone, i.e. the light cylinder radius, this emission may be modulated at both the spin and beat periods.

However, an attractive alternative situation also presenting itself occurs if the rotating magnetized white dwarf is an oblique rotator. Then the magnetosphere, outside the light cylinder radius $\left(r_{\text {lc }}\right)$, behaves like a relativistic wavy magnetic wind consisting of alternating regions of opposite polarity (see Fig. 8) due to the field contributions of both magnetic poles, i.e a "striped wind" ([20]).

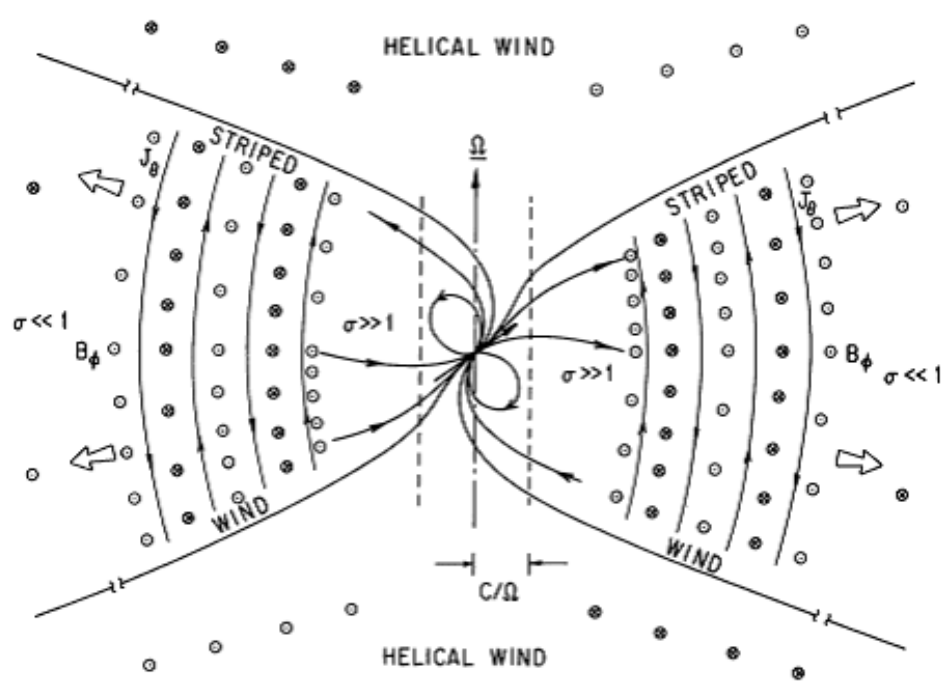

Figure 8: A representation of a relativistic "striped wind" consisting of regions of opposite magnetic polarity. Adopted from [20].

For these striped winds the inclination angle $\chi$ between the rotation and magnetic axes determine the amplitude of the zones of opposite magnetic polarity, e.g. see Fig 9 for a graphic representation of the helicity for two inclination angles, i.e $6^{\circ}$ (top) and $26^{\circ}$ (bottom) (e.g. [21]).

An attractive feature of the relativistic striped wind outside the light cylinder is that magnetic reconnection in the zones of opposite polarity can result in particle acceleration, and the synchrotron emission, even at moderate energies in the frame of the wind, can be Doppler boosted to high energy and will be pulsed (e.g. [22]). Particle acceleration in a striped wind scenario can occur through either current dissipation in the wind zone, or dissipation of the wind in a termination shock (e.g. [22]).

The condition for pulsed emission is that delay in Doppler boosted emission on a specific wave front beamed towards the observer (see geometry depicted in Fig. 10), i.e.

$$
\begin{aligned}
\Delta t & =\frac{\Delta R}{c} \\
& =\frac{(1-\cos \theta) R_{\circ}}{c}
\end{aligned}
$$

is less than the time delay between successive wave fronts, i.e. $\Delta T=(\Delta l / c)$. For highly relativistic flows, $\theta \sim(1 / \Gamma)<1$, where $\Gamma$ represents the bulk Lorentz factor of the wind, which 

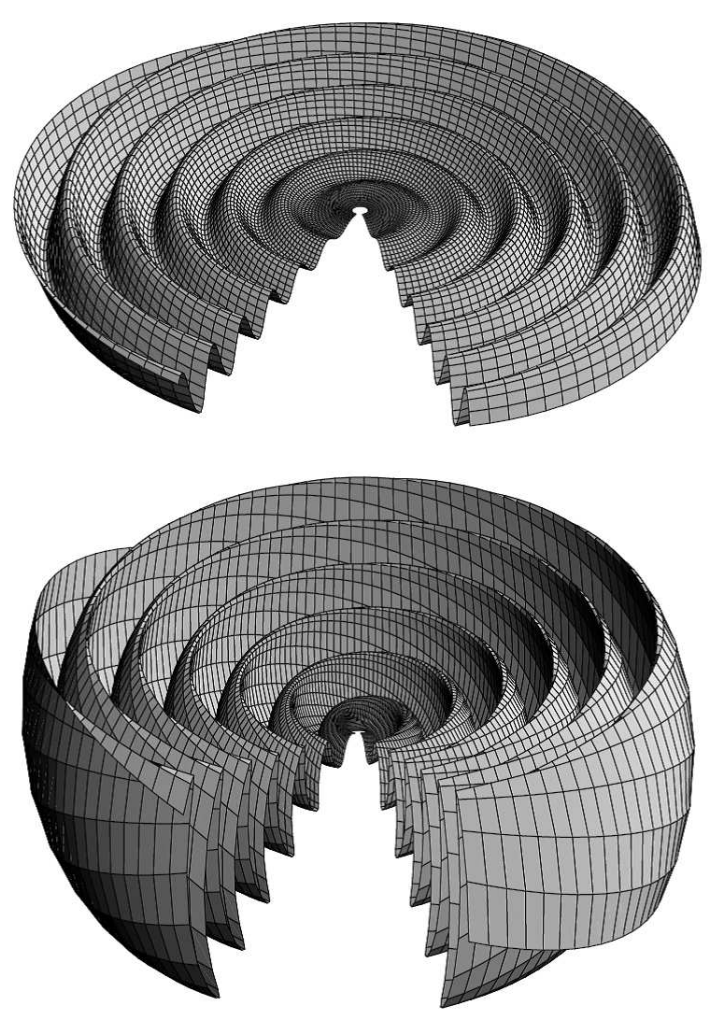

Figure 9: A representation of a relativistic "striped winds" for two different inclination angles $(\chi)$ between the magnetic and rotation axes, i.e. $6^{\circ}$ (top) and $26^{\circ}$ (bottom). Adopted from [21].

implies that

$$
\Delta t \approx \frac{R_{\circ}}{2 \Gamma^{2} c} .
$$

A consequence of this beamed emission on relativistic outward moving wave fronts is that pulsed emission at the rotation period of the pulsar is observed from a region with dimension

$$
R_{\circ}<2 \pi \Gamma^{2} r_{\mathrm{lc}} .
$$

which will still appear point-like from the observer's perspective. However, it has been shown earlier that the AR Sco binary system fits comfortably inside the light cylinder, which implies that the beat period will possibly be imprinted on this emission as well.

\section{The Evolution of AR Sco}

The short rotation period $\left(P_{\mathrm{s}} \approx 117 \mathrm{~s}\right)$ of the white dwarf in AR Sco suggests that the system perhaps evolved from a high mass transfer history where the white dwarf was spun-up to a period that is shorter than the current spin period of the white dwarf, similar to the evolution proposed for the novalike variable AE Aquarii $([23,24])$. The observed spin down of the white dwarf ([1]) 


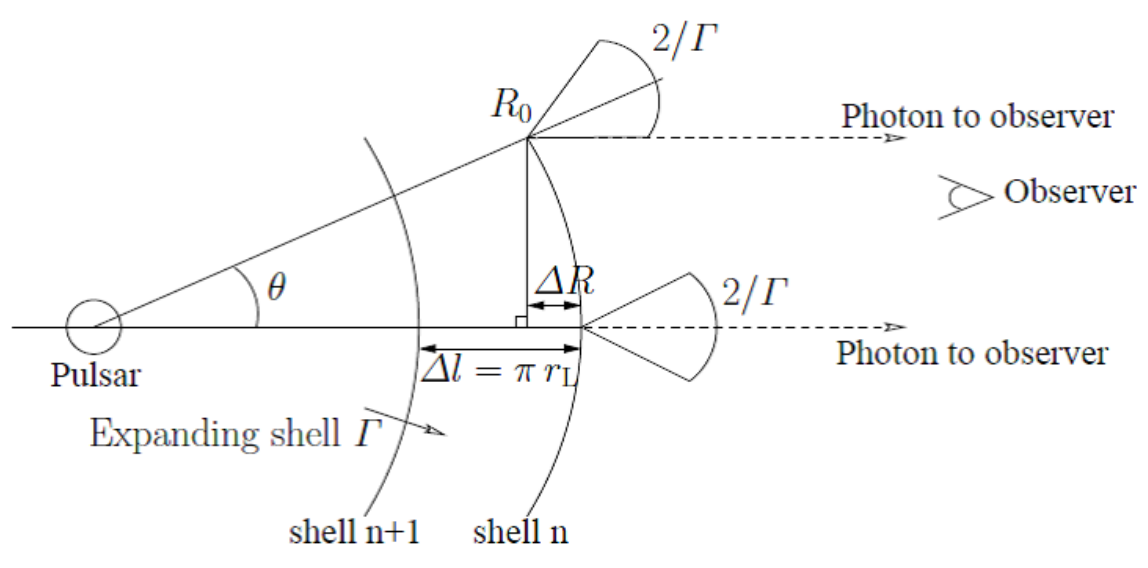

Figure 10: Doppler boosted emission towards the observer will be pulsed if the difference in light travel time between zones on the same wave front beamed towards the observer $(\Delta R)$ is less that the distance between two wave fronts $\Delta l=\pi r_{\mathrm{lc}}$ of different polarity. Adopted from [22].

infer a spindown time scale of approximately $\tau_{\mathrm{sd}} \sim 10^{7} \mathrm{yr}$ ([1]). The continuous interaction of the highly magnetic white dwarf with the magnetic secondary star, dissipates magnetic energy inside the envelope at a rate $\dot{W}=\left(B^{2} / 8 \pi\right)\left(4 \pi R_{2}^{2} \delta\right) \omega_{\mathrm{b}}$, where $B$ is the estimated magnetic field of the white dwarf at the distance of the secondary star, $\omega_{\mathrm{b}}$ is the beat period angular velocity of the white dwarf and where $\delta=\sqrt{2 \eta_{\text {tur }} / \omega_{\mathrm{b}}}$ represents the dissipation depth of magnetic energy, where $\eta_{\text {tur }}$ is the turbulent plasma resistivity (e.g. [2] and references therein). The dissipation of magnetic energy exerts a torque on the white dwarf $T_{\mathrm{D}}=\left(\dot{W} / \omega_{\mathrm{b}}\right)$ resuting in a synchronization timescale which is given by $t_{\mathrm{syn}}=I \omega_{\mathrm{b}} / T_{\mathrm{D}}$, where $I$ is the moment of inertia of the white dwarf, which is of the order of $I \sim 1.5 \times 10^{50} \mathrm{~g} \mathrm{~cm}^{2}$. If we assume a $500 \mathrm{MG}$ dipolar magnetic field for the white dwarf, the upper limit derived from the dipole radiation, the field strength at the distance of the secondary star is $B_{\mathrm{b} 1} \sim 160$ Gauss. For $\delta \sim 10^{8} \mathrm{~cm}$, i.e. using $\eta_{\text {tur }} \leq 10^{15} \mathrm{~cm}^{2} \mathrm{~s}^{-1}$ ([25]), the magnetic torque exerted on the secondary star is of the order $T_{\mathrm{D}}=\left(B_{1, *}^{2} / 8 \pi\right)\left(R_{\mathrm{wd}} / a\right)^{6}\left(4 \pi R_{2}^{2} \delta\right) \sim 10^{33} \mathrm{erg}$. The resulting spin-synchronization time scale for a $500 \mathrm{MG}$ white dwarf with the secondary star is of the order of $t_{\text {syn }} \sim 2.5 \times 10^{8}$ yr. Combined with the magnetic dipole radiation energy loss this synchronization time scale can in fact be shorter.

The eventual outcome of this synchronization process will be determined by the mass transfer evolution from the secondary star, which seem to be currently in a hibernation phase (e.g. [26]), perhaps after a nova eruption and severe mass outflow from the system driven by the large mass accretion that resulted in the spin-up of the white dwarf to a short rotation period. The onset of mass transfer from the secondary star over a time scale $t_{\mathrm{mt}}<t_{\mathrm{syn}}$ will perhaps drive the system towards an Intermediate Polar, whereas if $t_{\mathrm{mt}} \geq t_{\mathrm{syn}}$ the system may become a Polar.

\section{Conclusions}

The multi-frequency emission pulsar-like emission from AR Sco, from radio to X-rays frequencies, not driven by mass accretion, results in AR Sco probably being the prototype of a binary 
system white dwarf pulsar. The only other system that could possibly also be a member of this category, is the nova-like variable AE Aquarii. The multi-frequency emission of both these systems is driven by the spin-down of the white dwarf, which makes these systems unique among the close binaries and cataclysmic variables respectively. Although the conversion of spin-down energy to possible high energy gamma-ray emission would be rather low, both these sources may benefit form future studies with the Cherenkov Telescope Array (CTA), which will introduce a paradigm shift concerning sensitivity in the energy regime above $20 \mathrm{GeV}$.

\section{Acknowledgements}

The author appreciates the invitation to present this work at the conference as well as the hospitality he experienced at the Splendid Hotel La Torre in Mondello where the conference was hosted.

\section{References}

[1] T. R. Marsh et al. A radio-pulsing white dwarf binary star, Nature 537 (2016) 374

[2] D.A.H. Buckley, P.J. Meintjes, S.B. Potter, T.R. Marsh \& B.T. Gänsicke Polarimetric evidence of a white dwarf pulsar in the binary system AR Sco, Nature Astronomy 1 (2017) 0029

[3] S.B. Potter et al. Polarized QPOs from the INTEGRAL polar IGRJ14536-5522 (= Swift J 1453.4 - 5524), MNRAS 402 (2010) 1161

[4] B. Oruru \& P.J. Meintjes X-ray characteristics and the spectral energy distribution of $A E$ Aquarii, MNRAS 421 (2012) 1557

[5] W. Becker \& J. Trümper The $x$-ray luminosity of rotation powered neutron stars, $A \& \&$ $\mathbf{3 2 6}$ (1997) 682

[6] J.P Ostriker \& J.E. Gunn, On the nature of Pulsars I: Theory, ApJ 157(1969) 1395

[7] H. van der Laan, A model for variable extragalactic radio sources, Nature 211 (1966) 1131

[8] T.S. Bastian, G.A. Dulk \& G. Chanmugam Radio flares from AE Aquarii; A low power analog to Cyg X-3, ApJ 324 (1988) 431

[9] P.J. Meintjes \& L.A. Venter Modelling the continuous radio outbursts in AE Aquarii, MNRAS 341 (2003) 891

[10] J.-J Geng, B. Zhang \& Y.-F Huang A model of the white dwarf pulsar AR Scorpii, ApJ 831 (2016) L10

[11] J.L. Katz AR Sco, A white dwarf synchronar, ApJ (in press) (2017) [https://arxiv.org/abs/1609.07172]

[12] J. Takata, H. Yang \& K.S. Cheng A model for AR Scorpii: Emission from relativistic electrons trapped by closed magnetic fields of the white dwarf, ApJ 851 (2017) 143 
[13] J.-F Donati \& J.D. Landstreet Magnetic fields of nondegenerate stars, Ann. Rev. Astron. Astrophys. 47 (2009) 333

[14] L. Mestel \& H.C. Spruit On magnetic braking of late-type stars, MNRAS 226 (1987) 57

[15] C.G. Campbell Magnetohydrodynamics in Binary Stars, Kluwer Academic publishers, (1997) 88-89

[16] P.J. Meintjes \& E. Jurua Secondary star magnetic field in close binaries, MNRAS 372 (2006) 1279

[17] G. Haerendel Acceleration from field-aligned potential drops, ApJ Suppl. 90 (1994) 765

[18] P. Goldreich \& W.H. Julian Pulsar Electrodynamics, ApJ 157 (1969) 869

[19] J. Arons \& E.T. Scharlemann Pair formation above pulsar polar caps: structure of the low altitude accelerator zone, ApJ 231 (1979) 854

[20] F.V. Coroniti Magnetically striped relativistic magnetohydrodynamic winds: The Crab nebula revisited, ApJ 349 (1990) 538

[21] J. G. Kirk \& Y Lyubarski Reconnection in Pulsar Winds, Publications of the Astronomical Society of Australia 18 (2001) 415

[22] J. G. Kirk, Y Lyubarski \& J. Petri The theory of pulsar winds and nebulae, in Neutron Stars and Pulsars, Ed. W. Becker, Astrophysics and Space Science Library (Springer) 357 (2009) 421

[23] K. Schenker, A.R. King, U. Kolb, G.A. Wynn \& Z. Zhang AE Aquarii: how cataclysmic variables descend from supersoft binaries, MNRAS 337 (2002) 1105

[24] P.J. Meintjes On the evolution of the novalike variable AE Aquarii, MNRAS 336 (2002) 265

[25] P.J. Meintjes \& E. Jurua Secondary star magnetic fields in close binaries, MNRAS 372 (2006) 1279

[26] M. Shara, M. Livio, A.F.J. Moffat \& M. Orio Do novae hibernate during most of the millenia between eruptions? Links between dwarf and classical novae, and implications for the space densities and evolution of cataclysmic binaries, ApJ 372 (1986) 163 